Books, videos, CD-ROMs, DVDs and any

other relevant items submitted for a review

in the $B D J$ should be addressed to: Kate

Maynard, Assistant Editor, British Dental

Journal, NPG, 4-6 Crinan Street, London,

N1 9XW

\section{Dental public health: a primer}

\author{
M. Patel, N. Patel \\ UK: Radcliffe \\ price £21.95; pp 120 \\ ISBN 1857756479
}

Dental public health: a primer by Meera and Nakul Patel is an innovative and reader friendly book for undergraduate students who want an overview of dental public health. It will not nor is it intended to replace the conventional textbooks but it will serve students well as an introduction or an aide memoir for different aspects of the subject. The layout of the book is clever in that it appeals to a number of different types of learners through the use of punchy text, lists, illustrations, mnemonics, spider diagrams, tables and graphs. It has information crammed into seven easy to read colour coded chapters. What it lacks in detail it compensates for in accessibility. The seven sections cover Concepts of Dental Public Health, Epidemiology in Dentistry, Health Promotion, Oral Health Care, Preventive Dentistry, Terms and Definitions and Passing the Exams. Within the chapters each section has aims and objectives; the objectives are expressed as learning outcomes to indicate what the reader should be able to do having read the section. A question section at the end of every chapter allows the student to test themselves.

Each chapter presents a good overview of the subject and the reader is introduced to some acclaimed academics in the general health field, for example Illich, Dubos and McKeown. They are referred to under 'Ideas for Healthy Living'; this is a welcome inclusion as it serves as a signpost to an appropriate and fascinating broader literature, if the student is interested.

Within the section on Oral Health Care there is a well described and illustrated account of a student elective in India. There are very few textbooks with such graphic descriptions and in my opinion this will encourage students to go and see such practices themselves.

The chapter on preventive dentistry includes a section on smoking cessation which is an excellent addition to a book on dental public health and will reinforce the readers' perception of the role of the dental professional in helping patients to quit smoking. From a critical perspective, I was surprised that there was just one page dedicated to prevention of dental caries and that there was scant mention of fluoride toothpaste and other fluoride vehicles. Another issue is that the references are incomplete. Other than these concerns, I can safely recommend this book to undergraduate students as a companion to conventional texts. Congratulations to the authors and to those who taught and inspired them.

\section{H. Whelton}

\section{Contemporary orthodontics e-dition (fourth edition)}

\author{
W. R. Proffit, H. W. Fields Jr, D. M. Sarver \\ UK: Elsevier \\ price $£ 99.00$ \\ ISBN 0323046134
}

The majority of UK orthodontists will be familiar with earlier editions of this textbook but this new edition has taken a significant step forward with its use of an online facility.

Section 1 deals with malocclusion and dental deformity in contemporary society and details the prevalence of malocclusion in the population, its effects and also its relation to injury and disease. There is also a mention of IOTN and its limitations.

Section 2 deals with growth and development of the orthodontic problem and includes a section on CT scanning. Abnormal facial growth is well covered including aetiology of cleft lip and palate. The section on growth patterns in the dentofacial complex is particularly good with reference to growth rotations. There is also a section on facial growth in adults. Aetiology of orthodontic problems is well illustrated with references to local and genetic influences.

Section 3 covers orthodontic diagnosis and in keeping with previous editions describes the development of a 'problem list' approach to diagnosis which then leads to a specific treatment plan. The limitations of orthodontic treatment are discussed and the extraction/non-extraction debate is addressed.

Section 4 deals with treatment mechanics and the biological basis of orthodontic therapy. It discusses contemporary orthodontic appliances including the use of clear aligners such as Invisilign.

Section 5 covers treatment of preadolescent children including the role of functional appliances. The controversies of functional appliances are addressed and the references are comprehensive and contemporary.

Section 6 addresses the stages of comprehensive treatment in logical steps including an excellent chapter on retention. The book finishes off with a section on adult treatments and combined surgical and orthodontic treatment.

In the earlier sections in the book, there is widespread use of text boxes which summarise the key facts of the text for the reader. They are nicely highlighted and contribute to the clarity of the sections.

One of the main criticisms of any textbook is that they are out-of-date before they are published. Purchasers of this book will be able to access the book online and there is a section entitled Updates, which allows the reader to download text, and images that have been added since the book was published. The reader can also download 

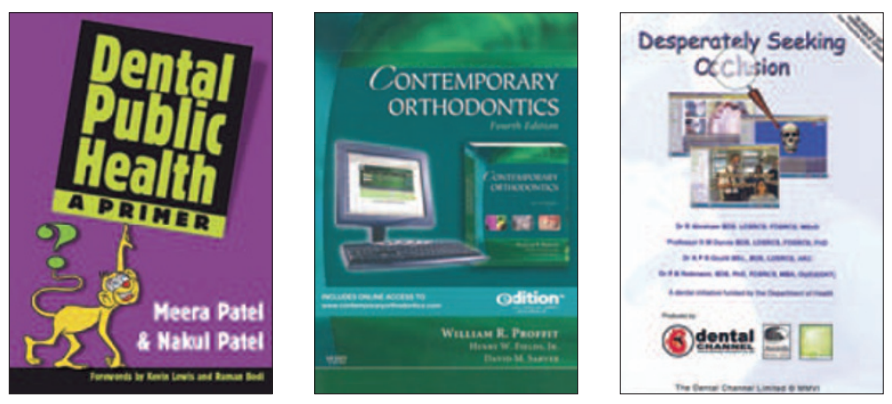

images from the book to use in lectures.

I feel the book would not be of interest to the general dental practitioner but is a must for all postgraduate orthodontic students and their teachers.

N. Fox

\section{Desperately seeking occlusion (CD-ROM)}

\section{Department of Health}

UK: Dental Channel

It is always flattering to be chosen to write an appraisal of one of the Dental Channel's products, but how my heart sank when I realised the topic in question was occlusion. Why couldn't it be crown and bridge or one of the more exciting aspects of our subject?

However, somehow the Dental Channel even manage to make the subject of occlusion interesting, and after all, any dental restoration we perform without a grasp of the principles of occlusion is likely to be problematic or fail prematurely.

One of the things I most like about the products we have seen over the years from the Dental Channel is that they stick fairly rigidly to a well tried and trusted format. Not only do we know that all is going to run fluently and proficiently, but because we have used other titles in the past, everything seems very familiar, and the time taken to become comfortable with the navigation of the package is minimal. The licensing process is identical in all packages from this stable, and I find that a quick email requesting an unlock code is answered and supplied in no time. Although the package may be used as a home study resource free of charge by dentists on the dental register, for those who really want to show off, you can even pay a relatively small fee and unlock the package in its verifiable format and get some inexpensive verified CPD.

On the home page the first four sections are the now familiar References, Case Studies, Quiz and Information and Links. The references section introduces the concepts of occlusion, and has five sections within it. There is a wealth of information here, and somehow it is put across in a succinct, yet unhurried way, with Andrew Gould's voice guiding you at every stage. In my view if you only studied this section alone you would learn a great deal about the subject matter. The terminology is all explained here, along with some of the dreaded anachronisms, but this information is a must for the rest of the learning experience. Additionally some clinical situations are looked at, and how you would put the principles of occlusion into use in the surgery. This includes both simple single broken tooth issues, right through to complex face bow registration.

The case studies section takes you through a number of cases involving both clinical and laboratory procedures. All are displayed with excellent clarity, using high quality video clips, and once again you are talked through each of the cases in a very clear fashion. These cases include a wide range of clinical scenarios and the necessary diagnostic tests and techniques which go along with the management of them. Prostheses including full dentures are looked at in this section, so it is not just restricted to the dentate mouth.

The Information and Links section tells you about the package and its use, but most importantly it invites you to join the growing list of people who have registered with the Dental Channel. Via the web you can contact the makers of the program and other likeminded people with a view to sharing ideas and comments.

The final section is the quiz. This allows the user to assess how much they know or don't know about the subject. It does allow you to assess your knowledge before and after the learning experience if you do the quiz before and after working through the $\mathrm{CD}$. It is also good in as much as it is easy to identify where you have knowledge gaps, and the material is here to fill them.

In conclusion I would like to congratulate the team at the Dental Channel on another excellent package to complement the titles we are all familiar with. New titles are being added to the range, and some old ones revised, so the website www.dental-channel.co.uk is worth keeping an eye on.

Sadly the Department of Health in their wisdom have decided to stop funding the production and distribution of this sort of CPD material, so we must all get used to the idea of funding some of our own CPD, but we should not let this put us off. How else may we extend or consolidate our knowledge without leaving our own chair, albeit the one in front of the computer?

D. Speechley 